\title{
Three-Dimensional Numerical Analysis of an Operating Helical Rotor Pump at High Speeds and High Pressures including Cavitation
}

\author{
Yang Zhou ${ }^{1, a}$, Lupeng $\mathrm{Wu}^{2}$ and Minghui $\mathrm{HAO}^{2}$ \\ ${ }^{1}$ School of Mechanical Engineering, Zhengzhou University,No. 100 Science Avenue, Zhengzhou,China \\ ${ }^{2}$ School of mechatronics Engineering, Harbin Institute of Technology, 92 West Da-zhi Street, Harbin, China
}

\begin{abstract}
High pressures, high speeds, low noise and miniaturization is the direction of development in hydraulic pump. According to the development trend, an operating helical rotor pump (HRP) at high speeds and high pressures has been designed and produced, which rotational speed can reach $12000 \mathrm{r} / \mathrm{min}$ and outlet pressure is as high as $25 \mathrm{MPa}$. Threedimensional simulation with and without cavitation inside the HRP is completed by the means of the computational fluid dynamics (CFD) in this paper, which contributes to understand the complex fluid flow inside it. Moreover, the influences of the rotational speeds of the HRP with and without cavitation has been simulated at $25 \mathrm{MPa}$.
\end{abstract}

\section{Introduction}

Gear pumps are widely applied in aerospace hydraulic system, chemical industry and civil engineering for the reason of its reliability, simple structure and low cost. Cavitation and trapped-oil are two major limitations to the development of high pressures, high speeds and of gear pumps. Due to trapped-oil, the gear meshing pressure increases suddenly, which dramatically decreases the lifespan of gears. Unfortunately, the vibration and noise are caused by trapped-oil so the stability of pump system drops down[1-2]. In this case, HRP, a kind of special gear pump, becomes relatively popular because it has no trapped-oil and flow ripples, low noise as well[3]. However, cavitation is the other non-ignorable limitation. Besides it can produce pressure fluctuations and noise, it also has effect on volumetric efficiencies[4-7].Along with the cavitation, the problems of two-phase flow appear, which have significant influences on work performance of gear pumps[8]. Numerical simulation of an external gear pumps operating at speeds below 3000rpm including cavitation is studied by D. del Campo[4], who discusses the influences on the volumetric efficiency under different rotational speeds. In order to understand the complex fluid dynamic characterization, Zhou et al[9] simulate 2D gear pumps running at high pressures and high speeds, but cavitation is not considered. Thus,HRP is used to eliminate the effect of trapped-oil in this paper. In order to predict the actual movement of the internal flow inside the HRP, whose three-dimensional simulations including cavitation at high speeds (10000rpm and $12000 \mathrm{rpm})$ and high pressure $(25 \mathrm{Mpa})$ are finished. Furthermore, the influences on the meshing pressure and the maximum negative pressure at different rotational speeds are studied.

\footnotetext{
${ }^{\mathrm{a}}$ Corresponding author : rumsfld@126.com
} 


\section{Mesh model of HRP}

The HRP has no trapped-oil for the reason of its special tooth profile. Two rotors mesh in the HRP, as depicted in Figure1. When the driving rotor spin clockwise, the hydraulic oil trapped in the inter-teeth volumes between rotors and body is moved from the inlet to the outlet by rotors. In this way, the pressure is formed and lifted in high pressure chamber. The transverse contact ratio of the HRP is 0.5 so that rotors keep contacting in one-point. The design of the tooth profile studied by Zhou et al[10] has been referenced to establish the mathematical model of rotors in this paper. As an example, the mathematical model of driven rotor is schematically shown in Fig.1.

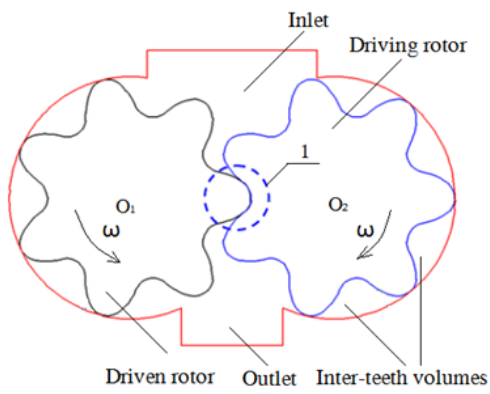

Figure 1. The tooth profiles of the HRP

The tooth profile of the HRP consists of two equal circular arcs and one involute. The mathematical equation for the circular arc and as well as the involute can be obtained with the method presented by Zhou et al[10]. The computational domain of the HRP has been divided into three regions, this is schematically shown in Fig. 2. That consists of inlet chamber, outlet chamber and rotoring zone.

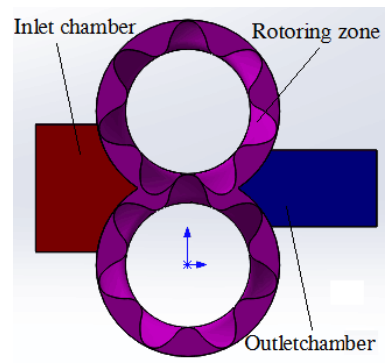

Figure 2. Computational domain of the HRP

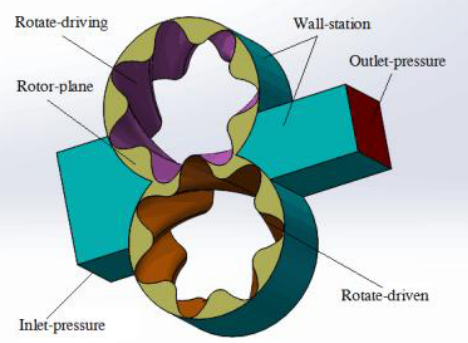

Figure 3. Boundary conditions

Fig. 3 shows a detail of boundary conditions ANSYS ICEM CFD is used to create meshes which formed by tetrahedral cells, as shown in Fig. 4. In order to improve the mesh quality, avoiding the errors of negative meshes and non-convergence in the process of simulations, several methods are applied as follows. Triangular prism grid is used to detail the edges of rotoring zone.Meshes whose quality below 0.3 are deleted.The irrelevant grid points are removed.Finally, 1085141 cells are obtained from the computational domain, as depicted in Fig. 4. 

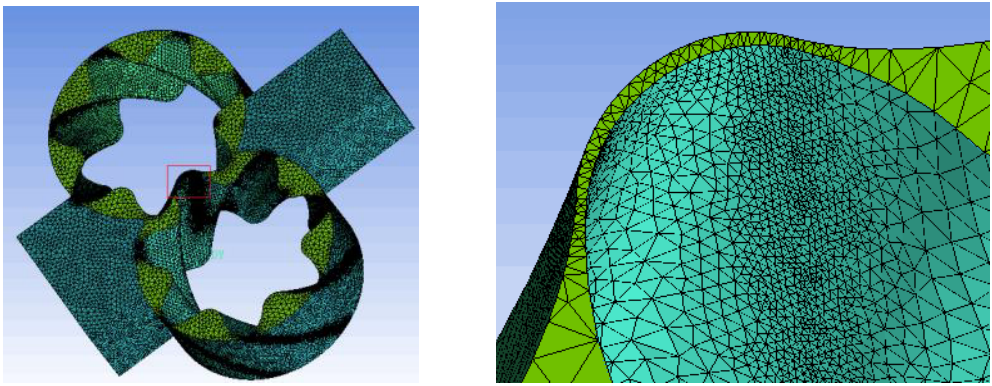

Figure 4. 3D mesh and detail of rotoring zone

Both geometrical parameters of the HRP and properties of mineral oil are presented in table1. The commercial finite-volume-based code ANSYS Fluent 16.0 has been applied in numerical simulations of the HRP.

Table 1. Geometrical parameters of pump and properties of mineral oil

\begin{tabular}{|c|c|}
\hline Length of the inlet chamber & $22.15 \mathrm{~mm}$ \\
\hline Width of the inlet chamber & $15 \mathrm{~mm}$ \\
\hline Length of the outlet chamber & $17.85 \mathrm{~mm}$ \\
\hline Width of the outlet chamber & $9 \mathrm{~mm}$ \\
\hline Height of the rotors & $10 \mathrm{~mm}$ \\
\hline Modulus & $2 \mathrm{~mm}$ \\
\hline Number of teeth & 7 \\
\hline Diameter of rotors & $21.334 \mathrm{~mm}$ \\
\hline Distance between centers of rotors & $18.08 \mathrm{~mm}$ \\
\hline Pressure angle & $28^{\circ}$ \\
\hline Density & $960 \mathrm{~kg} / \mathrm{m} 3$ \\
\hline Dynamic viscosity & $0.048 \mathrm{~Pa} \cdot \mathrm{s}$ \\
\hline
\end{tabular}

In order to illustrate that the cells number have no influence on the results of the simulation, the computational domain of the HRP is divided into two groups with different number of cells. The former contains 580329 cells, the later contains 1085141 cells. In the process of simulation, the remeshing algorithm do not stop changing the number of cells in each time step until remeshing is reached. The number of cells of grids in both two groups stabilized around 880 thousands after several meshing cycles. This can be seen in Fig.5. Considering the speed of calculation and quality of grids, the latter is selected in the end.As far as author knows, numerical simulation including cavitation requires more strict convergence criteria than that without it. Singhal et al model has been selected in this paper. This model is attractive because it can solve the continuity and the momentum equation for the mixture. The first phase chooses fuel-oil-liquid while air is used in the second phase. The parameters of the cavitation model are $F_{V a p}=0.4$ and $F_{\text {cond }}=0.001$. 


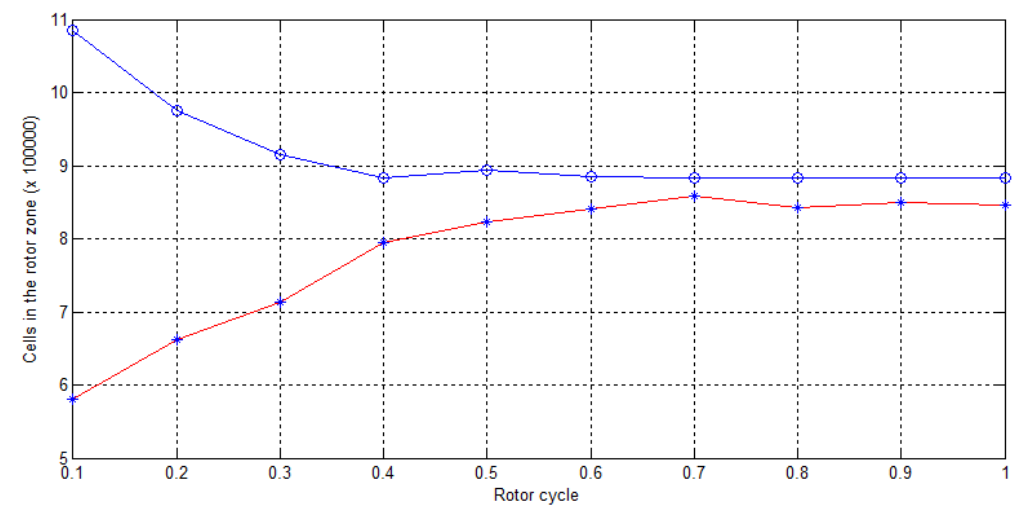

Figure 5. Cells number evolution in computational domain

\section{Results of the numerical simulation}

Regarding three-dimensional numerical simulations, several methods proposed in this paper allow the author to study the complicated flow inside the HRP. In this case, effects without and with cavitation at different rotational speeds are studied, which can predict the performance of the HRP operating at high pressure and high speeds, although it can not represent the actual values. The pressures of the flow inside the HRP are simulated when it operates at $25 \mathrm{MPa}$ and $10000 \mathrm{rpm}$. After $0.0048 \mathrm{~s}$, the contours of pressure are shown in Fig. 6.

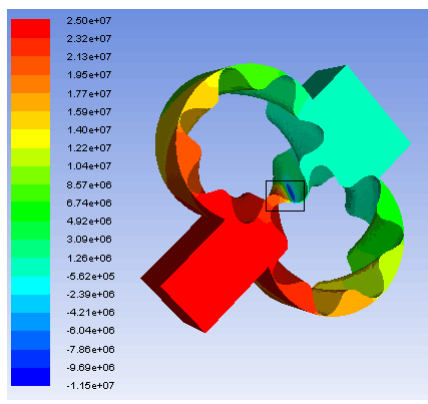

(a)

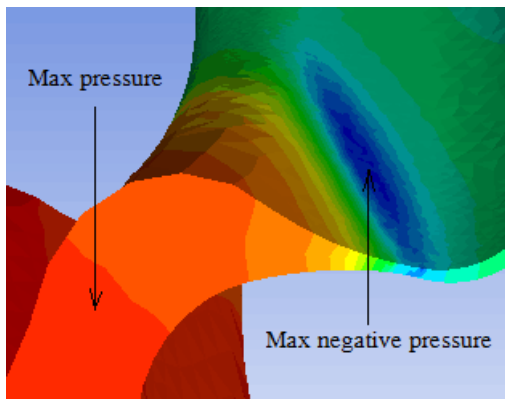

(b)

Figure 6. Contours of pressure and pressure where rotors mesh

In Fig.6(a), from inlet chamber to outlet chamber, the closer to the outlet, the pressure higher. This is because the hydraulic oil trapped in the inter-teeth volumes between rotors and body is moved from the inlet to the outlet by rotors. The maximum pressure appears in the region that is close to the contact point of two rotors in outlet chamber. The maximum negative pressure is found in inlet chamber, as depicted in Fig. 6(b). The pressure distribution is similar to the one obtained from 2D simulation [8], however, the results of 3D simulation are more straightforward. Interestingly, the maximum negative pressure reaches $16 \mathrm{MPa}$ in $3 \mathrm{D}$ simulation.

Effects without and with cavitation at different rotational speeds are studied when the outlet pressure is assumed at $25 \mathrm{MPa}$. Fig. 7 compares the effects on the maximum pressure without and with cavitation. Meshing pressures gradually increase with the higher rotational speeds. However, the minimum of the maximum pressure appears at 0.8 rotor cycle and this condition is not affected by rotational speeds. Cavitation has little effect on the maximum pressures because it mainly exists in low pressure chamber. Nevertheless, when considering cavitation, the maximum pressures increase 
nearly $4 \mathrm{MPa}$ at $10000 \mathrm{rpm}$. The same figure shows that influence of cavitation on the maximum pressure exists mainly from 0.3 to 0.7 rotor cycle.
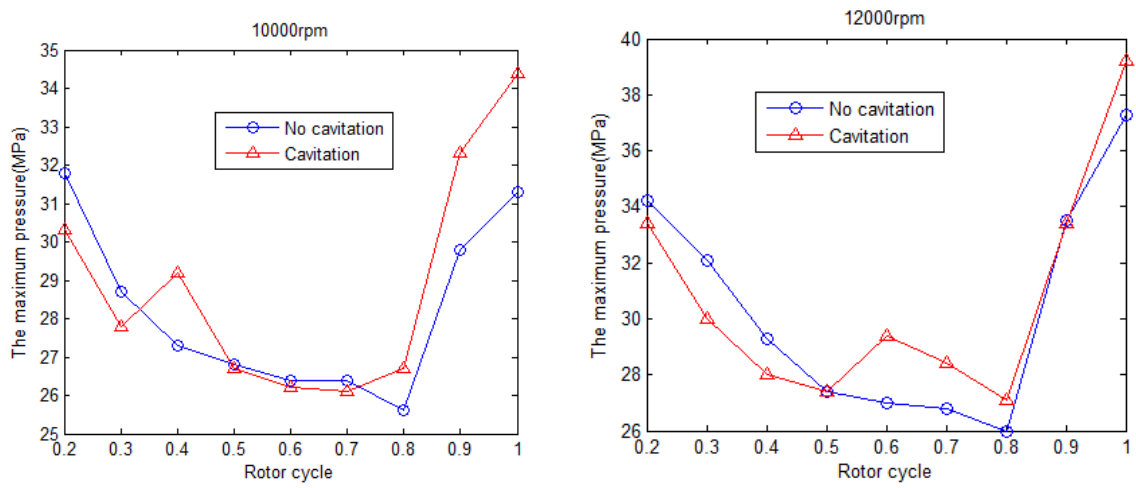

Figure 7. Analysis of the maximum pressure with and without cavitation at 10000rpm and 12000rpm.
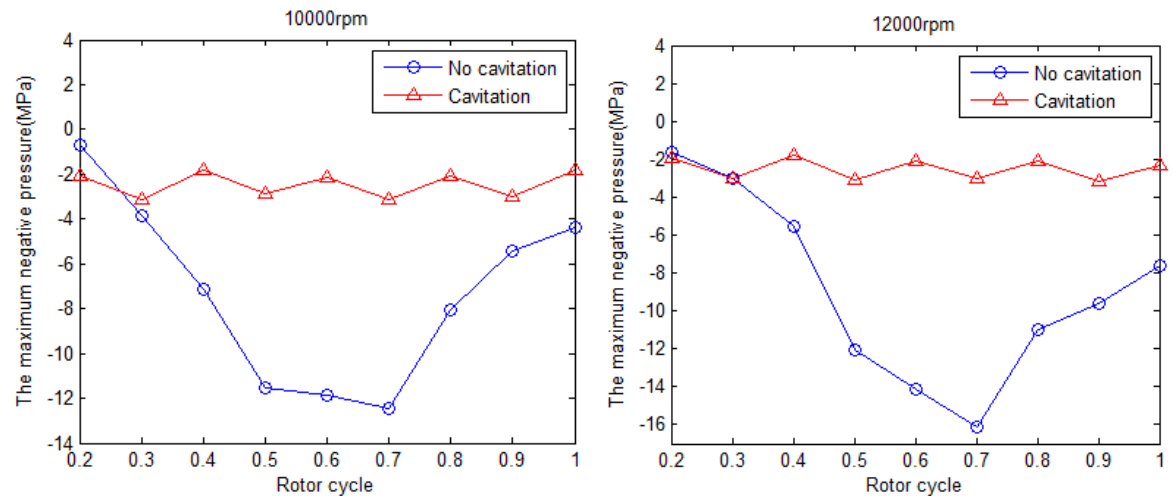

Figure 8. Analysis of the maximum negative pressure with and without cavitation at $10000 \mathrm{rpm}$ and $12000 \mathrm{rpm}$

In Fig.8, the effects on the maximum negative pressure without and with cavitation at different rotational speeds are studied. No considering cavitation, the decline of the maximum negative pressures become more urgent with the increase of the rotational speeds and at 0.7 rotor cycle, where the maximum negative pressures reach the lowest points. In this case, the pressure difference between the maximum pressure and the maximum negative pressure becomes larger, which leads to more leakage, and the stability of rotors decreases as well. The maximum negative pressure reaches nearly $16 \mathrm{MPa}$ when the HRP operates at $12000 \mathrm{rpm}$. The negative pressure fluctuations are significantly smaller when considering cavitation. Through the whole rotor cycle, the maximum negative pressures maintain around $-2 \mathrm{MPa}$, since cavitation mainly exists in low pressure chamber.

\section{Conclusions}

Three-dimensional numerical analysis of the HRP has been proposed by means of computational fluid dynamics (CFD). Furthermore, cavitation has been considered, which contributes to obtain more accurate results. The results of simulation do not equal the actual conditions. However, it contributes to study structure optimization and seal of the HRP. Comparisons of effects on the maximum pressures and the maximum negative pressures at 10000rpm and $12000 \mathrm{rpm}$ are presented in this paper. The pressure fluctuations where rotors mesh inside the HRP are obvious. The maximum 
pressure and the maximum negative pressure as the rotational speeds become higher. Cavitation has little effect on the maximum pressure where rotors mesh. However, the low pressure chamber is filled with cavitation, by which a part of leakage is prevented.

\section{References}

1. Eaton M, Keogh PS and Edge KA. The Modelling, prediction, and experimental evaluation of gear pump meshing pressures with particular reference to aero-engine fuel pumps. Proc IMechE Part I: J Systems and Control Engineering.220,396-404,(2006).

2. Shu W, Sakurai H. and Aditya K. The Optimal Design in External Gear Pumps and Motors. IEEE/ASME Transactions on Mechatronics 16, 945-952,(2011).

3. Lee G S, Jung SY and Bae JH. Design of rotor for internal gear pump using cycloid and circulararc curves. Journal of Mechanical Design. 1-12,134(1),(2012). .

4. Del campo D, Castilla R, Raush GA, et al. Numerical analysis of external gear pumps including cavitation. ASME J Fluids Eng.1-9,134,(2012).

5. Neyrat S, Orand $\mathrm{N}$ and Jonquet D. Modeling and analysis of an automatic transmission internal gear oil pump with cavitation. SAE 2005 Noise and Vibration Conference and Exhibition,16-19, (2005).

6. Andrea Vacca, Richard Klop and Monika Ivantysynova. A numerical approach for the evaluation of the effects of air release and vapour cavitation on effective flow rate of axial piston machines. International journal of fluid power ,33-45, 11(1),(2010).

7. Abbot, Philip A., Walsh, James. And Halas, Richard. Cavitation noise investigation of a pumpturbine. Waterpower '91: A New View of Hydro Resources.2031-2040,(1991).

8. Heath R A. Theoretical analysis of cavitation conditions in gear pumps. Internal Report B60, 080, Lucas Aerospace Ltd, Birmingham, 1977.

9. Yang Zhou, Shuanghui Hao and Minghui Hao. A two-dimensional numerical analysis of a circular-arc gear pump operating at high pressures and high speeds. Proc IMechE Part E: J Process Mechanical Engineering ,1-12,(2015).( online).

10. Yang Zhou, Shuanghui Hao and Minghui Hao. Design and performance analysis of a circulararc gear pump operating at high pressure and high speed. Proc IMechE Part C: J Mechanical Engineering Science.189-20.230,(2016). 